\title{
Analytical Investigation of Magnetic Field on Unsteady Boundary Layer Stagnation Point Flow of Water-Based Graphene Oxide-Water and Graphene Oxide-Ethylene Glycol Nanofluid over a Stretching Surface
}

\author{
Ali Rehman and Zabidin Salleh \\ Department of Mathematics, Faculty of Ocean Engineering Technology and Informatics, Universiti Malaysia Terengganu, \\ Kuala Nerus 21030, Terengganu, Malaysia \\ Correspondence should be addressed to Zabidin Salleh; zabidin@umt.edu.my
}

Received 24 September 2020; Revised 3 February 2021; Accepted 15 March 2021; Published 23 March 2021

Academic Editor: Hussein Abulkasim

Copyright (c) 2021 Ali Rehman and Zabidin Salleh. This is an open access article distributed under the Creative Commons Attribution License, which permits unrestricted use, distribution, and reproduction in any medium, provided the original work is properly cited.

\begin{abstract}
This study explains the effect of magnetic field of the stagnation point flow of a water-based nanofluid graphene oxide-water (GO$\mathrm{W}$ ) and graphene oxide-ethylene glycol (GO-EG). Heat transfer analyses are discussed by converting the given partial differential equation into a nonlinear ordinary differential equation using the similarity transformation and solved using an approximate analytical method, namely, the optimal homotopy analysis method (OHAM), to obtain an approximate analytical solution of the nonlinear problem that analyzes the problem. The BVPh 2.0 package function of Mathematica is used to obtain the numerical results. The results of important parameters such as the magnetic field parameter, unsteady parameter, stretching parameter, Prandtl number, Eckert number, and kinematic parameter for both velocity and temperature profiles are plotted and discussed. The convergence control parameter of the approximate analytical method is obtained up to the 25th iteration using the BVPh 2.0 package. The skin friction coefficient and Nusselt number are explained in tabular form.
\end{abstract}

\section{Introduction}

In the history of fluid mechanics, the derivation of the boundary layer equation and its solution using similarity transformation is an important area for researchers. With the help of boundary layer theory, both Newtonian and non-Newtonian fluids can be modeled. The results obtained using the boundary layer theory are more similar to the experimental results. The industrial application of nonNewtonian fluids is higher than that of Newtonian fluids because of the use of non-Newtonian fluids in petroleum drilling polymer engineering and manufacturing of food and paper. Recently, the more active area of graphene oxide as a nanofluid is a rotating disk model problem. In a hexagonal lattice, graphene is a single layer of carbon atoms. Because graphene is a single layer of carbon and graphene oxide is a two-dimensional material, compared with graphene, researchers are interested in graphene oxide. In 1859, graphene oxide was introduced by $\mathrm{Sir}$ Second Baronent Benjamin Colline using the chemical method, Brodie, through the oxidation of bulk graphite with potassium chlorate and nitric acid. Lerf and Klinowsky used a modified Hummers' method to study the natural structure of graphene oxide. Graphene oxide has some key applications in engineering and industry, such as computer storage systems, turbomachinery hard disk jet motors, and turbine systems. It is known that the thermal conductivity of the base fluid is lower than that of the nanofluid, and to increase the heat transfer ratio, a different method is used; for example, the reduced heat transfer time and heat exchanger size can be minimized. It is known that the thermal conductivity of ethylene glycol is greater than that of the base fluid; therefore, ethylene glycol is used as a cooling fluid and antifreezing agent. 
Many nonlinear relations were observed for the stress and the rate of strain for non-Newtonian fluids. It is difficult to express all the properties of a non-Newtonian fluid in a single equation. The flow owing to the stretching sheet of the boundary layer of non-Newtonian fluids has important applications in several manufacturing processes, such as molten polymers through a slit die for the production of plastic sheets, hot rolling wire and fiber coating, processing of food stuffs, metal spinning glass fiber production, and paper production. In mechanical applications, non-Newtonian fluid models play an important role. Numerous materials have incredible significance in ventures; for example, liquid plastic, nourishment items, divider paint, oils, lubricant oils, and penetrating mud have non-Newtonian fluid conduct. According to scientists, non-Newtonian liquids are the most pertinent to exploration. One of the precarious subclasses is Sisko fluid, which has more centrality as an event in many designing activities. The common sense application has delivered an enthusiasm for examining the feasibility of differential conditions overseeing streams of non-Newtonian fluids, which have various uses in the field of design, applied mathematics, and computer engineering. Munir et al. [1] used an extended sheet to study the bidirectional stream of the Sisko fluid. Olanrewaju et al. [2] used a level plate to study the Sisko precarious free convective liquid stream in the presence of heat exchange. Khan et al. [3] considered the Sisko fluid, which is consistent with the heat movement in an annular pipe. Khan et al. [4] used an extended surface to study the Sisko limit-layer liquid course. Patel et al. [5] studied a laminar Sisko fluid limit stream. Darji et al. [6] studied the Sisko fluid precarious common convective limit-layer stream. Siddiqui et al. [7] proposed a Sisko fluid film on a vertical belt for a waste framework.

Khan et al. [8] used an annular pipe to study the Sisko fluid. Sar et al. [9] reported the limit-layer conditions of a Sisko fluid. Marinca et al. [10] examined Maxwell fluid courses using permeable extending plates. Moallemi et al. [11] discovered a definite answer for the Sisko fluid stream in a pipe. Hayat et al. [12] investigated Maxwell nanofluids. Malik et al. [13] investigated an Erying-Powell nanofluid through an extension of the blended convective stream of magnetohydrodynamics (MHD). Nadeem et al. [14] investigated Maxwell fluid transfer through a vertical extending surface in the event of nanoparticles. Raju et al. [15] investigated an MHD nano non-Newtonian fluid through a cone with free convective heat and mass exchange. Rokni et al. [16] investigated a nanoliquid stream with heat exchange through plates. Nadeem et al. [13] investigated the numerical arrangement of a nanofluid stream on an extended sheet. Shehzad et al. [17] investigated the Jaffrey nanofluid MHD stream in the event of convective limit situations. Sheikholeslami et al. [18] used a nanofluid stream with an attractive field in the presence of heat exchange. Mahmoodi et al. [19] investigated a nanofluid stream with heat exchange for cooling applications. Recently, Shah et al. $[20,21]$ investigated nanofluid streams with the effects of heat radiation and lobby current with a turning framework. The most recent theoretical study and investigation of
Sheikholeslami on nanofluids utilizing disparate marvels with current application, assets, and properties with the use of different methodologies can be considered in [22-25]. Rehman et al. [26] studied analytically the Marangoni convection of a thin-film spray of CNT nanofluids using a stretching cylinder. In this study, the effect of a magnetic field on the unsteady boundary layer stagnation point flow of a water-based nanofluid graphene oxide-water (GO-W) and graphene oxide-ethylene glycol (GO-EG) with stretching surfaces is explained. Rehman et al. [27] studied analytically a thin-film unsteady nanofluid using a stretching sheet. Gul et al. [28] discussed the performance of graphene oxide nanofluids flowing in an upright channel through a permeable medium. Gul et al. [29] discussed the impact of Marangoni and thermal radiation convection on a graphene-oxide-water-based and ethylene-glycol-based nanofluid. Next, Gul et al. [30] discussed integer and noninteger order studies of GO-W and GO-EG nanofluids. Gul et al. [31] used rotating disks to study the stable dispersion of a graphene oxide nanofluid analytically.

In this paper, we will explain the approximate analytical solution of the unsteady boundary layer stagnation point flow of water-based GO-W and GO-EG nanofluids with stretching surfaces in the presence of a magnetic field, and variable thermal conductivity is obtained. The given partial differential equation is converted to a nonlinear ordinary differential equation using the similarity transformation and solved using the approximate analytical method (OHAM). Liao et al. [32] used this method for the first time and showed that the convergence control parameter of this method is faster than the approximate solution of the nonlinear problem as compared with other analytical methods. Using this method, we found a series solution of the velocity and temperature profiles. The impacts of different parameters on both the velocity and temperature profiles were plotted and discussed.

\section{Mathematical Formulation}

Let us assume an unsteady two-dimensional incompressible flow of nanofluids over a stretching sheet. The stretching sheet velocity in $x$ direction is denoted by $U_{\varpi}=b x / 1-\gamma t$, where $b$ and $\gamma$ are constants, both of which are non-negative and $t$ represents time. $B_{0}$ represents a uniform magnetic field, which was assumed from the Faradays. $B(t)$ represents the magnetic field and is defined as $B(t)=B_{0}(1-\gamma t)^{-(1 / 2)}$, which depends on time $t$, where $T_{\circledast}(x, t)=T_{0}-T_{r}\left(b x^{2} / 2 \nu\right)(1-\gamma t)^{-(3 / 2)}$ represents the temperature distribution on the surface that changes in the $x$ direction (Figure 1).

Under the above conditions, the boundary layer equation for unsteady flow toward the stretching surface can be written as follows:

$$
\begin{gathered}
\frac{\partial u}{\partial x}+\frac{\partial v}{\partial y}=0 \\
\frac{\partial u}{\partial t}+u \frac{\partial u}{\partial x}+v \frac{\partial u}{\partial y}=U_{s} \frac{d U_{s}}{d x}+v \frac{\partial^{2} u}{\partial y^{2}}-\frac{\sigma B_{0}^{2}}{\rho_{n f}}
\end{gathered}
$$




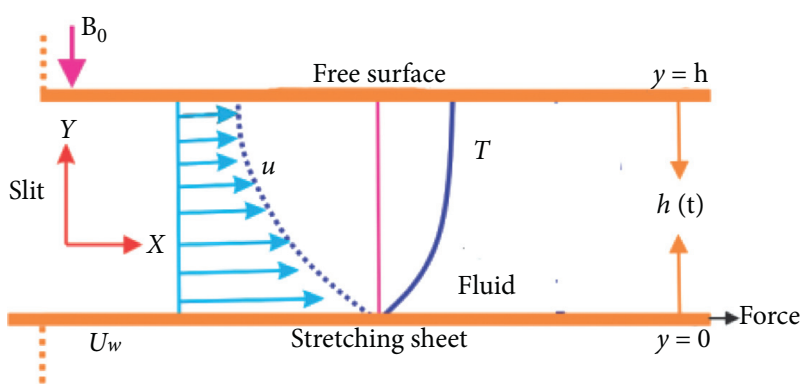

Figure 1: Geometry of the problem.

The temperature distribution in the flow field is as follows:

$$
\frac{\partial T}{\partial t}+u \frac{\partial T}{\partial t}+v \frac{\partial T}{\partial t}=\frac{k}{\rho C_{p}} \frac{\partial^{2} T}{\partial y^{2}}+\frac{\mu_{s}}{\left(\rho C_{p}\right)_{f}}\left(\frac{\partial u}{\partial y}\right)^{2} .
$$

The temperature is denoted by $T, k$ represents the thermal conductivity, $\rho$ is the fluid density, $C_{p}$ is the specific heat, and the dynamic viscosity of the nanofluid is denoted by $\mu_{s} . u$ and $v$ are the velocity components along the $x$ and $y$ directions, respectively, $x$ denotes the distance along the sheet, $y$ represents the distance perpendicular to the sheet, the velocity of the stagnation point is denoted by $U_{s}=a x$ with $a>0$, where $v$ represents the kinematic viscosity of the fluid, the magnetic field is denoted by $M$ and is defined as $M=\sigma B_{0}^{2} / \rho_{n f}$, the unsteady parameter is denoted by $S$ and is defined as $S=\gamma / b$, Eckert number is defined as Ec $=U_{\varpi}^{2} / C_{p}\left(T_{\varpi}-T_{0}\right)$, and $A=b / c$ is the stretching parameter.

The boundary condition for velocity component is given as follows:

$$
u=U_{w}, v=0 \text { at } y=0 ; u \longrightarrow U_{s} \text { as } y \longrightarrow \infty .
$$

The stretching velocity of the sheet is denoted by $U_{w}=c x$, where $c$ is the stretching constant. The boundary conditions for the temperature distribution were as follows:

$$
T=T_{w} \text { at } y=0 ; T \longrightarrow T_{\infty} \text { as } y \longrightarrow \infty .
$$

In equation (5), $T_{w}$ represents the temperature at the sheet and $T_{\infty}$ represents the free stream temperature, which is assumed to be constant. The stream function is defined as follows:

$$
u=\frac{\partial \psi}{\partial y}, v=-\frac{\partial \psi}{\partial x}
$$

The similarity transformation is defined as follows:

$$
\begin{aligned}
\psi & =\sqrt{\frac{a \nu_{f}}{1-\alpha t}} x f(\eta), \eta=y \sqrt{\frac{a}{\nu_{f}(1-\alpha t)}}, \text { and } \\
\theta(\eta) & =\frac{T-T_{\infty}}{T_{w}-T_{\infty}} .
\end{aligned}
$$

The similarity transformation from equation (7) is used in equations (2) and (3) to convert the PDE into a nonlinear ordinary differential equation.

$$
\begin{aligned}
& f^{\prime \prime \prime}-(1-\phi)^{2.5}\left((1-\phi)+\phi \frac{\rho_{s}}{\rho_{f}}\right) f^{\prime \prime} f-f^{\prime 2} \\
& -s(1-\phi)^{2.5} M\left(\frac{\eta}{2} f^{\prime \prime}+f^{\prime}\right)=0,
\end{aligned}
$$

with boundary condition $f(\eta)=0$ and $f^{\prime}(\eta)=c / a$ at $\eta=0$, and

$$
\frac{k_{n f}}{k_{f}} \theta^{\prime \prime}-\operatorname{Pr}\left((1-\phi)+\phi \frac{\left(\rho C_{p}\right)_{s}}{\left(\rho C_{p}\right)_{f}}\right)\left(\theta^{\prime} f\right)-\frac{s}{2} \operatorname{Ec}\left(\eta \theta^{\prime}\right)=0
$$

with boundary condition $\theta(\eta)=1$ at $\eta=0 ; \theta(\eta) \longrightarrow 0$ as $\eta \longrightarrow \infty$.

The skin friction coefficient $C_{n f}$ is defined as $C_{n f}=2 \tau_{\Phi} / \rho U_{\varpi}^{2}$, and the local Nusselt number $N u_{x}=\left(q_{\Phi} / k\right.$ $\left.\left(T_{\oplus}-T_{0}\right)\right) x$.

\section{Method of Solution}

The nondimensionless equations (8) and (9) are solved analytically by an approximate analytical method, namely, the OHAM, which is given as follows:

$$
L(u(x))+N(u(x))+g(x)=0, B(u(x)),
$$

where $L$ represents a linear operator, $x$ represents an independent variable, $g(x)$ represents an unknown function, $N$ represents a nonlinear operator, and $B(u)$ represents a boundary operator. Using this method, we first obtain a family of equations:

$$
\begin{aligned}
H(\phi(x), p)= & (1-p)[L,(\phi(x, p))+g(x)] \\
& -H(p)[L(\phi(x, p))+g(x) \\
& +N(\phi(x, p))]=0, \\
B(\phi(x, p))= & 0,
\end{aligned}
$$

where $p$ represents an embedding parameter and lies in $[0,1], H(p)$ represents a nonzero auxiliary function for $p \neq 0$ and $H(0) \neq 0$, and $\phi(x, p)$ is an unknown function. The initial estimates obtained from equations (8) and (9) are as follows:

$$
\begin{aligned}
f_{0}(\eta) & =\frac{c}{a} \eta, \\
\theta_{0}(\eta) & =1, \\
L_{f} & =\frac{d^{3} f}{d \eta^{3}}, \\
L_{\theta} & =\frac{d^{2} \theta}{d \eta^{2}},
\end{aligned}
$$

with constant properties

$$
L_{f}\left(C_{1}+C_{2} \eta+C_{3} \eta^{2}+C_{4} \eta^{3}\right)=0, L_{\theta}\left(C_{5}+C_{6} \eta\right)=0 .
$$


For equations (7) and (8), the average squared residual error is obtained from the following equation:

$$
\begin{aligned}
& \varepsilon_{m}^{f}=\frac{1}{n+1} \sum_{j=1}^{n}\left[K_{f}\left(\sum_{j=1}^{n} f(\eta)_{\eta=j \delta \eta}\right)\right], \\
& \varepsilon_{m}^{\theta}=\frac{1}{n+1} \sum_{j=1}^{n}\left[K_{\theta}\left(\sum_{j=1}^{n} f(\eta)_{\eta=j \delta \eta}, \sum_{j=1}^{n} \theta(\eta)_{\eta=j \delta \eta}\right)\right], \\
& \varepsilon_{m}^{t}=\varepsilon_{m}^{f}+\varepsilon_{m}^{\theta} .
\end{aligned}
$$

\section{Result and Discussion}

This section explains the consequences of different nondimensional parameters such as kinematic viscosity, magnetic field, stretching parameter, Prandtl, Eckert number, and unsteady parameter on velocity and temperature distribution $(\nu, M, A, \operatorname{Pr}, \mathrm{Ec}$, and $S$ ). The thermophysical properties of the base fluid and nanofluid are presented in Tables 1 and 2 , respectively. The influence of skin friction and Nusselt number is presented for both GO-W and GO-EG in Tables 3 and 4 . Table 3 shows that the skin friction coefficient is a decreasing function of the magnetic parameter and unsteady parameter; that is, by increasing the value of the magnetic parameter and the unsteady parameter, the skin friction coefficient decreases in both GO-W and GO-EG. Table 4 shows that the Nusselt number coefficient is a decreasing function of the Prandtl number and Eckert number; that is, higher values of the Prandtl number and Eckert number decrease the Nusselt number coefficient in both GO-W and GO-EG. Tables 5 and 6 show the convergence of the given approximate analytical method for both GO-W and GO-EG nanofluids up to the 25th iteration, and it is observed from Tables 5 and 6 that with an increase in the number of iterations, a strong convergence is attained. Figure 2 shows the influence of the magnetic field parameters on the velocity profile. The relationship between $f^{\prime}(\eta)$ and $M$ is inversely related. The velocity profile is a decreasing function of the magnetic field parameter, that is, a higher value of the magnetic field parameter decreases the velocity profile. With the increase in magnetic field resistance, certain types of forces known as Lorentz force are produced, which oppose the motion of the fluid particles in both GO-EG and GO-W; therefore, the velocity profile decreases, as presented in Figure 2. Figure 3 shows the influence of the unsteady parameters on the velocity profile. The values of $f^{\prime}(\eta)$ and $M$ were inversely related. The velocity profile is a decreasing function of the unsteady parameter; that is, a higher value of the unsteady parameter decreases the velocity profile. By increasing the unsteady parameter, the motion of the fluid particles in both GO-EG and GO-W decreases, resulting in a decrease in the velocity profile, as shown in Figure 3. The influence of the stretching parameter on the velocity profile is shown in Figure 4 . The relation between the velocity field $f^{\prime}(\eta)$ and the stretching parameter $A$ is direct. The velocity is the increasing function of the stretching parameter; that is, the increasing values of the stretching parameter $A$ increase
TABle 1: Thermo physical properties.

\begin{tabular}{lccc}
\hline Physical properties & $\begin{array}{c}\text { Thermal conduct } \\
(\mathrm{kW} / \mathrm{mK})\end{array}$ & $\begin{array}{c}\text { Specific heat } \\
(\mathrm{Cp} / \mathrm{kg} \mathrm{K})\end{array}$ & $\begin{array}{c}\text { Density } \\
\left(\rho \mathrm{kg} / \mathrm{m}^{3}\right)\end{array}$ \\
\hline Base fluid water & 0.135 & 2190 & 753 \\
GO-W & 6500 & 2400 & 2500 \\
GO-EG & 3000 & 1700 & 1500 \\
\hline
\end{tabular}

TABLE 2: Thermal conductivity values at different volume fractions.

\begin{tabular}{lccccc}
\hline Volume fraction $\varphi$ & 0.0 & 0.01 & 0.02 & 0.03 & 0.04 \\
\hline Knf (GO-W) & 0.135 & 0.137 & 0.214 & 0.215 & 0.256 \\
Knf (GO-EG) & 0.135 & 0.162 & 0.3 & 0.218 & 0.247 \\
\hline
\end{tabular}

TABle 3: Comparison of the skin friction for the two nanofluids when $\operatorname{Pr}=15.6, v=0.9$, and $A=0.1$.

\begin{tabular}{lccc}
\hline$S$ & $M$ & $f^{\prime \prime}(0)$ GO-W $\phi=0.01$ & $f^{\prime \prime}(0)$ GO-EG $\phi=0.01$ \\
\hline 0.7 & 1 & 0.929705 & 0.39471 \\
0.8 & & 0.92164 & 0.379547 \\
0.9 & & 0.91147 & 0.37264 \\
& 2 & 0.9100877 & 0.36994 \\
& 3 & 0.77608 & 0.33724 \\
& & 0.768432 & 0.3246 \\
& & 0.75818 & 0.31641 \\
\hline
\end{tabular}

TABLE 4: Comparison of the Nusselt number $\left(\operatorname{Re}_{x}^{-(1 / 2)} N u_{x}\right)$ for the two nanofluids when $v=0.5, M=2$, and $A=0.7$.

\begin{tabular}{cccc}
\hline $\operatorname{Pr}$ & Ec & $f^{\prime \prime}(0)$ GO-W $\phi=0.01$ & $f^{\prime \prime}(0)$ GO-EG $\phi=0.01$ \\
\hline 3 & 5 & 0.51231 & 0.49077 \\
5 & & 0.49341 & 0.48237 \\
7 & & 0.47451 & 0.47397 \\
& 7 & 0.45614 & 0.45647 \\
& 9 & 0.43776 & 0.44897 \\
& & 0.42795 & 0.43121 \\
& & 0.35021 & 0.41346 \\
\hline
\end{tabular}

TABle 5: Convergence of the method for GO-EG when $\operatorname{Pr}=6, M=10, \mathrm{Ec}=1, v=1$, and $A=1$.

\begin{tabular}{lcc}
\hline$m$ & $\varepsilon_{m}^{f}$ GO-EG & $\varepsilon_{m}^{\theta}$ GO-EG \\
\hline 5 & $1.36438 \times 10^{-1}$ & $2.86775 \times 10^{-1}$ \\
10 & $7.14094 \times 10^{-3}$ & $1.48738 \times 10^{-2}$ \\
15 & $5.209443 \times 10^{-7}$ & $1.07298 \times 10^{-4}$ \\
20 & $4.37298 \times 10^{-9}$ & $8.54131 \times 10^{-5}$ \\
25 & $3.95787 \times 10^{-11}$ & $7.94423 \times 10^{-6}$ \\
\hline
\end{tabular}

TABLE 6: Convergence of the approximate analytical method for GO-W when $\operatorname{Pr}=6, M=10, \mathrm{Ec}=1, v=1$, and $A=1$.

\begin{tabular}{lcc}
\hline$m$ & $\varepsilon_{m}^{f} \mathrm{GO}-\mathrm{W}$ & $\varepsilon_{m}^{\theta} \mathrm{GO}-\mathrm{W}$ \\
\hline 5 & $1.07991 \times 10^{-1}$ & $2.88574 \times 10^{-1}$ \\
10 & $5.65266 \times 10^{-2}$ & $1.0759 \times 10^{-3}$ \\
15 & $4.12383 \times 10^{-3}$ & $1.0759 \times 10^{-5}$ \\
20 & $3.4616 \times 10^{-4}$ & $8.55721 \times 10^{-7}$ \\
25 & $3.133 \times 10^{-5}$ & $8.006632 \times 10^{-9}$ \\
\hline
\end{tabular}




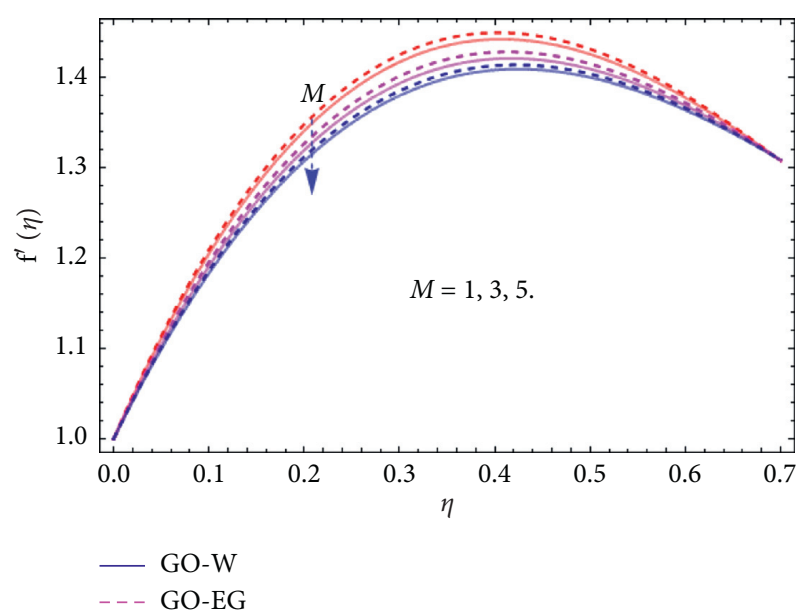

Figure 2: Effect of magnetic field on velocity profile.

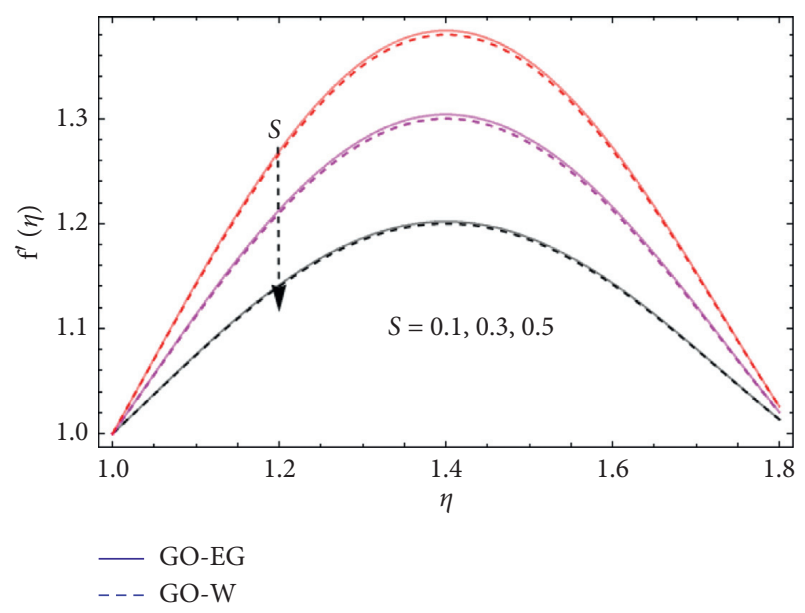

FIGURE 3: Effect of unsteady parameter on velocity profile.

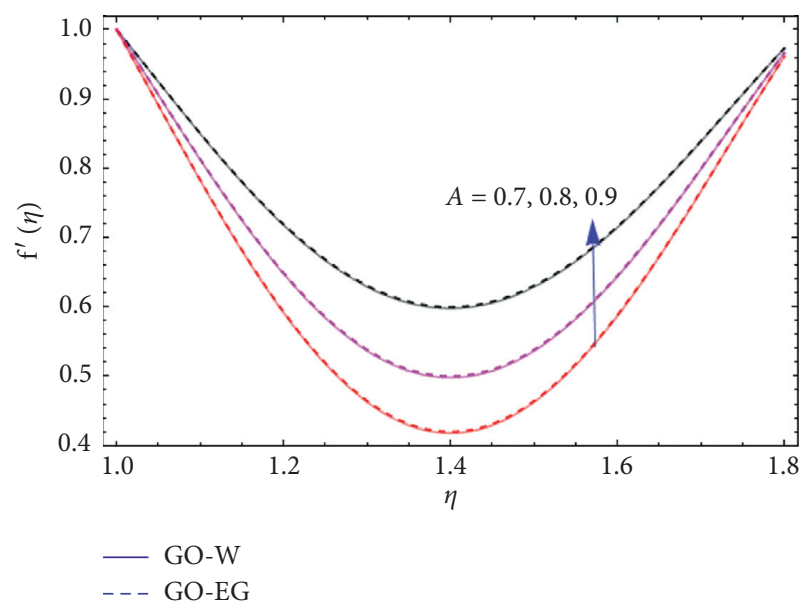

FIGURE 4: Effect of stretching parameter on velocity profile.

the velocity field $f^{\prime}(\eta)$. Physically, by increasing the stretching parameter $A$, the position of the fluid particle changes; thus, the moment of the particle increases, and the velocity field is enhanced by increasing the stretching

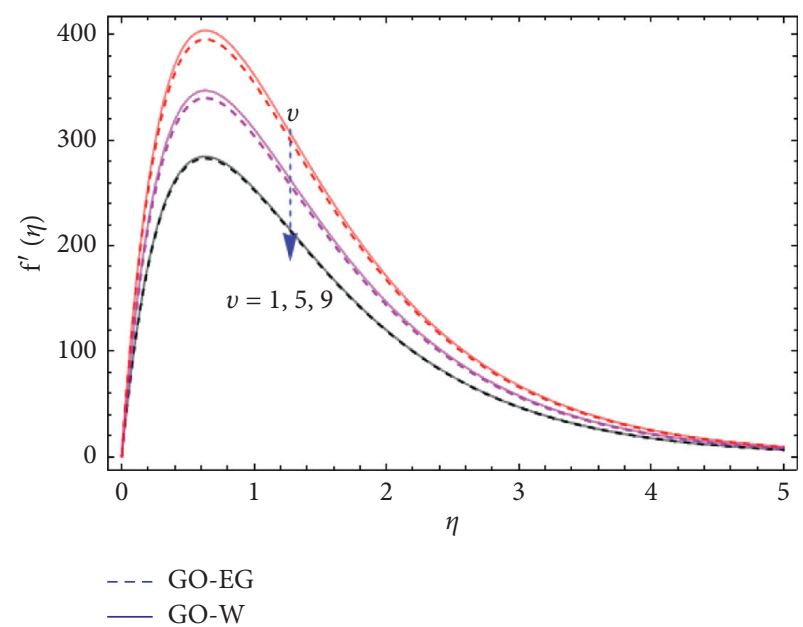

FIGURE 5: Effect of kinematic viscosity on velocity profile.

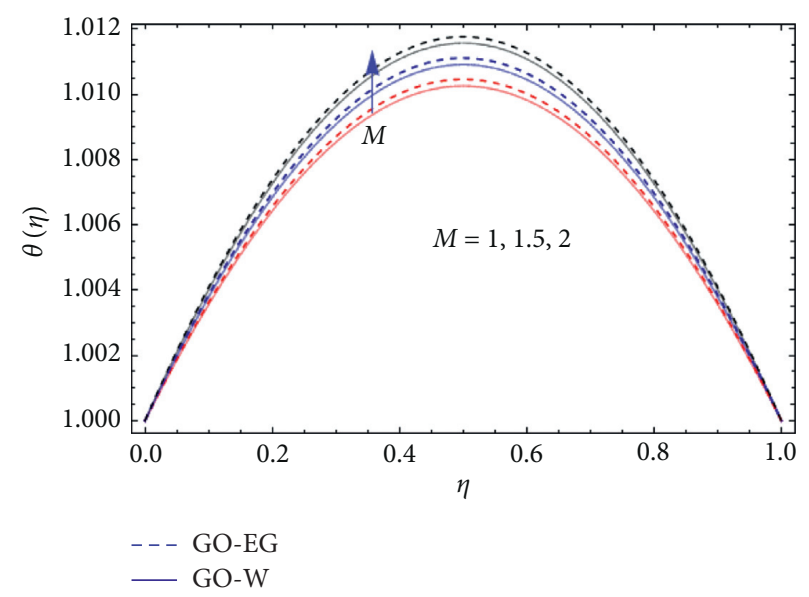

FIGURE 6: Effect of magnetic field on temperature profile.

parameter $A$, as shown in Figure 4. The influence of the kinematic viscosity on the velocity profile is shown in Figure 5. The relationship between the velocity profile and kinematic viscosity is inverse, or the velocity profile is a decreasing function of the kinematic viscosity; that is, higher values of the kinematic viscosity $\alpha$ decrease the velocity profile $f^{\prime}(\eta)$, as shown in Figure 5. By increasing the kinematic viscosity, viscous forces that oppose the motion of fluid particles in both GO-EG and GO-W are produced; therefore, the velocity profile decreases with increasing kinematic viscosity. Figure 6 shows the effect of the magnetic field on the temperature profile. It can be observed that by increasing the magnetic field, the temperature profile increases or the temperature profile is a decreasing function of the magnetic parameter. Such a state occurs because of the increase in resistance forces, which opposes the motion of fluid particles in both GO-EG and GO-W, resulting in friction forces that increase the temperature profile. The influence of the Prandtl number on the temperature profile is shown in Figure 7. From Figure 7, it can be observed that the temperature profile is a decreasing function of the Prandtl number; that is, a higher value of Pr decreases the 


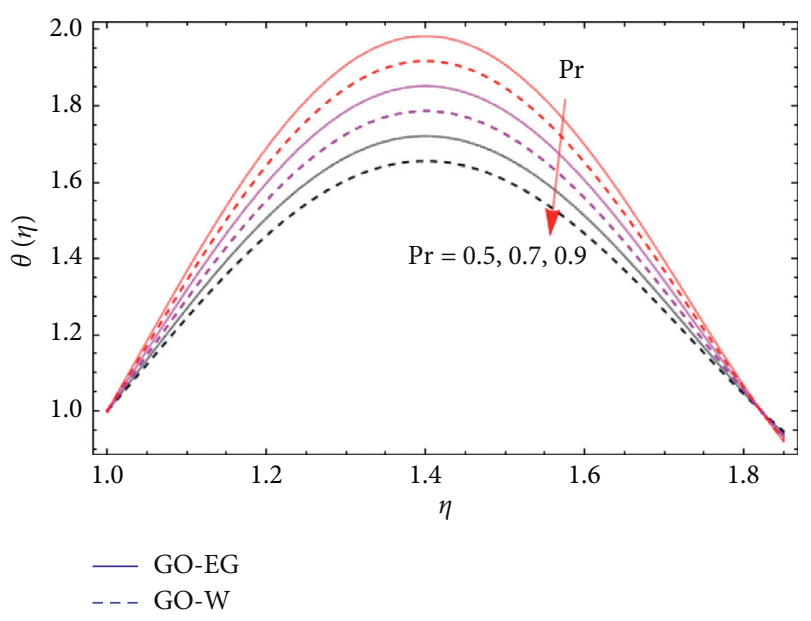

Figure 7: Effect of Prandtl number on temperature profile.

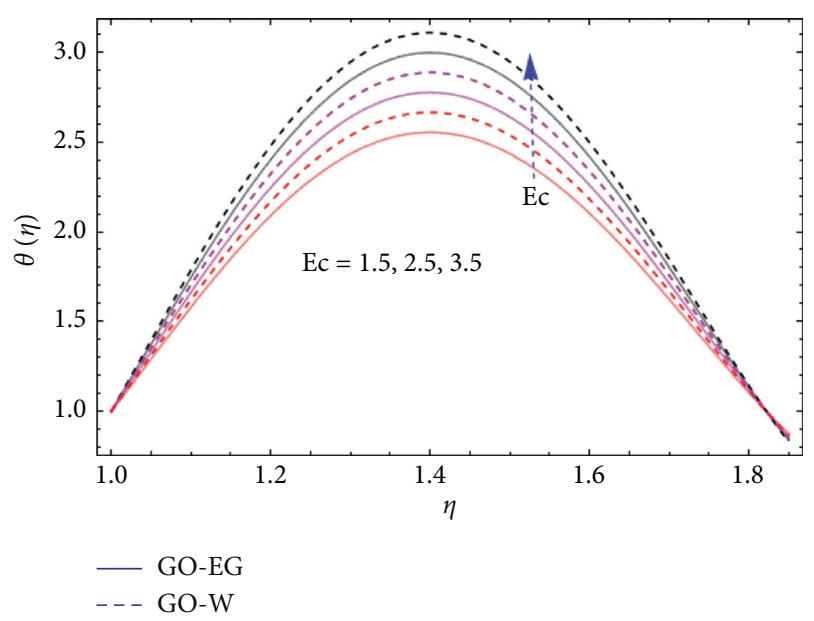

Figure 8: Effect of Eckert number on temperature profile.

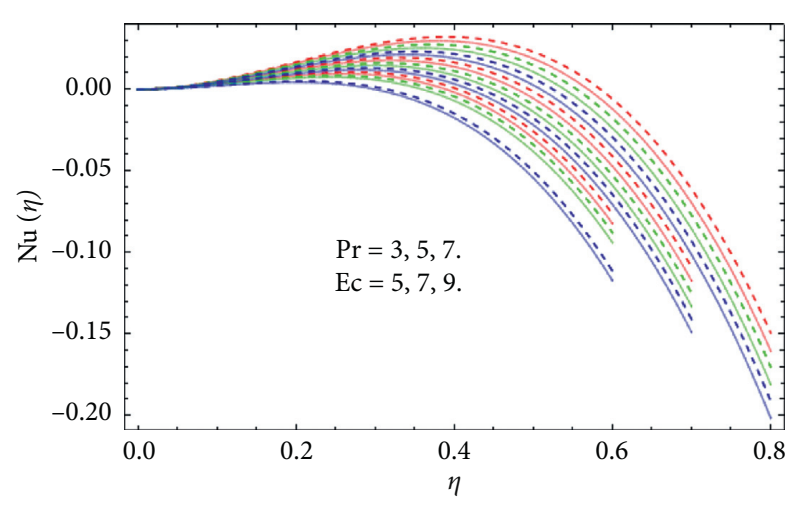

FIgURE 9: Comparison of the Nusselt number for the two nanofluids.

temperature profile in both GO-EG and GO-W, as presented in Figure 7. This effect is because the thickness of the momentum boundary layer is greater than that of the thermal boundary layer, and as a result, a higher value of the Prandtl number decreases the thermal boundary layer. The

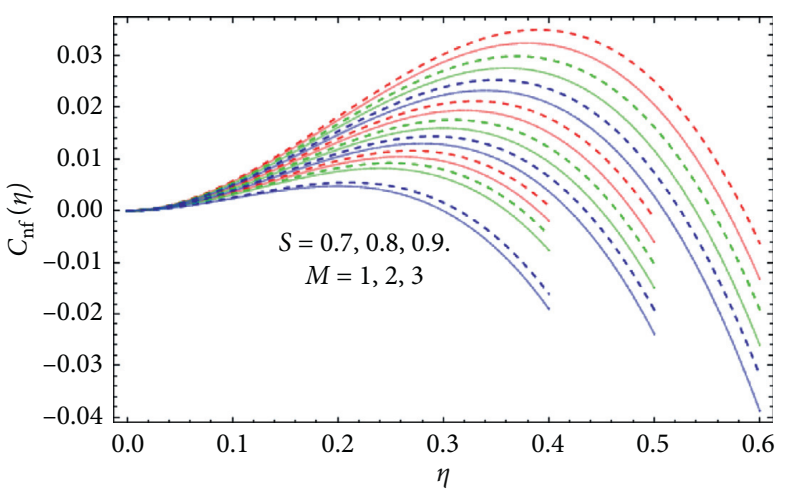

FIGURE 10: Comparison of the skin friction for the two nanofluids.

influence of the Eckert number on the temperature profile is shown in Figure 8. From Figure 8, it can be seen that the temperature profile is an increasing function of the Eckert number; that is, the increasing value of Eckert number increases the temperature field, and thermal diffusion is higher viscous diffusion; therefore, the improved value of Ec increases the temperature profile. The relationship between Nusselt number, Prandtl number, and Eckert number is shown in Figure 9. We see that Nusselt number is the decreasing function of Prandtl number and Eckert number in both nanofluids. Figure 10 shows the relationship between skin friction coefficient, unsteady parameter, and magnetic field parameter. We see that skin friction coefficients are the decreasing function of unsteady parameter and $M$ (magnetic field parameter) in both nanofluids.

\section{Conclusion}

In this paper, we explain the approximate analytical solution of the unsteady boundary layer stagnation point flow of water-based nanofluids GO-W and GO-EG with stretching surfaces in the presence of a magnetic field, and variable thermal conductivity is obtained. The given partial differential equation is converted to a nonlinear ordinary differential equation by using the similarity transformation and solved by an approximate analytical method, namely, the OHAM. The practical application of this study is to produce cooling and heating effects, which also have significant applications in engineering and industrial machinery; the key objective of which is the circular pressure gradient and stability of centrifugal forces. The numerical results were obtained using the BVPh 2.0 package function of Mathematica. The results of important parameters such as the magnetic field parameter, unsteady parameter, stretching parameter, Prandtl number, Eckert number, and kinematic parameter were obtained. The effects of the skin friction coefficient and Nusselt number coefficient are explained in table form. The outputs obtained were as follows:

(i) The velocity profile is the decreasing function of the unsteady parameter $S$; that is, it decreases the velocity field by increasing the unsteady parameter

(ii) The velocity profile is an increasing function of the stretching parameter $A$; that is, the velocity profile 
increases with an increase in the stretching parameter

(iii) The temperature profile is the decreasing function of the Prandtl number; that is, the temperature profile decreases with the increasing value of the Prandtl number

(iv) The temperature profile is an increasing function of the Eckert number; that is, the temperature profile increases with increasing Eckert number

(v) The velocity profile is a decreasing function of the magnetic field $M$; that is, the velocity profile decreases with an increase in the magnetic field $M$ [33-35]

\section{Nomenclature}

$S: \quad$ Unsteady parameter

$C_{p}: \quad$ Specific heat

$\mu_{n f}$ : Dynamic viscosity of the nanofluid axial directions

$W_{w}$ : $\quad$ Stretching velocity

$\rho_{n f}: \quad$ Density of the nanofluids

$\eta$ : $\quad$ Similarity variable

Ec: $\quad$ Eckert number

$\varphi$ : $\quad$ Solid particle volume fraction

Q: $\quad$ Heat generation/absorption parameter

$\sigma^{*}: \quad$ Stefan-Boltzmann constant

$v_{n f}: \quad$ Kinematic viscosity of the nanofluids

$C_{f}: \quad$ Skin friction

T: $\quad$ Local temperature

$\delta: \quad$ Thickness of the liquid film

Pr: $\quad$ Prandtl number

$U_{w}$ : Suction, injection velocity

$T_{\delta}$ : Temperature at the free surface

$k^{*}$ : Mean absorption coefficient

$k^{*}$ : $\quad$ Mean absorption coefficient

$q_{r}: \quad$ Thermal radiative heat flux

$\beta$ : $\quad$ Nondimensional thickness of the film liquid

$k_{n f}$ : Thermal conductivity of the nanoparticles

$N u$ : Nusselt number

$\beta_{n f}$ : Thermal expansion coefficient of solid particles

$(u, w)$ : Velocity components.

\section{Data Availability}

No data were used to support this study.

\section{Conflicts of Interest}

The authors declare that they have no conflicts of interest for the publication of this paper.

\section{Acknowledgments}

This research was partially supported by the Universiti Malaysia Terengganu under the Postgraduate Research Grant (PGRG) vote no. 55193/3. The authors would like to thank Editage (http://www.editage.com) for English language editing.

\section{References}

[1] M. Khan, S. Munawar, and S. Abbasbandy, "Steady flow and heat transfer of a Sisko fluid in annular pipe," International Journal of Heat and Mass Transfer, vol. 53, no. 7-8, pp. 1290-1297, 2010.

[2] M. Khan and A. Shahzad, "On boundary layer flow of a Sisko fluid over a stretching sheet," Quaestiones Mathematicae, vol. 36, no. 1, pp. 137-151, 2013.

[3] M. Patel, J. Patel, and M. G. Timol, "Laminar boundary layer flow of Sisko fluid," Applications and Applied Mathematics: An International Journal, vol. 10, no. 2, pp. 909-918, 2015.

[4] R. M. Darji and M. G. Timol, "Similarity analysis for unsteady natural convective boundary layer flow of Sisko fluid," International Journal of Advances in Applied Mathematics and Mechanics, vol. 13, pp. 22-36, 2014.

[5] A. M. Siddiqui, H. Ashraf, T. Haroon, and A. Walait, "On the analytic solution for the steady drainage of magnetohydrodynamic (MHD) Sisko fluid film down a vertical belt," International Journal of Ayurveda \& Alternative Medicine, vol. 8, pp. 465-480, 2017.

[6] M. Khan, Q. Abbas, and K. Duru, "Magnetohydrodynamic flow of a Sisko fluid in annular pipe: a numerical study," International Journal for Numerical Methods in Fluids, vol. 62, pp. 1169-1180, 2010.

[7] G. Sar, M. Pakdemirli, T. Hayat, and Y. Aksoy, "New perturbation iteration solutions for Fredholm and Volterra integral equations," Journal of Applied Mathematics, vol. 2013, Article ID 682537, 9 pages, 2013.

[8] V. Marinca, R.-D. Ene, B. Marinca, and R. Negrea, "Different approximations to the solution of upper-convected Maxwell fluid over a porous stretching plate," Abstract and Applied Analysis, vol. 2014, Article ID 139314, 12 pages, 2014.

[9] N. Moallemi, I. Shafieenejad, and A. B. Novinzadeh, "Exact solutions for flow of a Sisko fluid in pipe," Bulletin of the Iranian Mathematical Society, vol. 37, pp. 49-60, 2011.

[10] S.-J. Liao, "An explicit, totally analytic approximate solution for Blasius' viscous flow problems," International Journal of Non-Linear Mechanics, vol. 34, no. 4, pp. 759-778, 1999.

[11] S.-J. Liao, "On the analytic solution of magnetohydrodynamic flows of non-Newtonian fluids over a stretching sheet," Journal of Fluid Mechanics, vol. 488, pp. 189-212, 2003.

[12] M. Y. Malik, K. Imad, H. Arif, and T. Salahuddin, "Mixed convection flow of MHD Eyring-Powell nanofluid over a stretching sheet," Neural Computing \& Applications, vol. 5, pp. 117-118, 2015.

[13] S. Nadeem, R. U. Haq, and Z. H. Khan, "Numerical solution of non-Newtonian nanofluid flow over a stretching sheet," Applied Nanoscience, vol. 4, no. 5, pp. 625-631, 2014.

[14] S. Nadeem, R. U. Haq, and Z. H. Khan, "Numerical study of MHD boundary layer flow of a Maxwell fluid past a stretching sheet in the presence of nanoparticles," Journal of the Taiwan Institute of Chemical Engineers, vol. 45, no. 1, pp. 121-126, 2014.

[15] C. S. K. Raju, P. Sanjeevi, M. C. Raju, S. M. Ibrahim, G. Lorenzini, and E. Lorenzini, "The flow of magnetohydrodynamic Maxwell nanofluid over a cylinder with Cattaneo-Christov heat flux model," Continuum Mechanics and Thermodynamics, vol. 29, no. 6, pp. 1347-1363, 2017.

[16] H. B. Rokni, D. M. Alsaad, and P. Valipour, "Electrohydrodynamic nanofluid flow and heat transfer between two plates," Journal of Molecular Liquids, vol. 216, pp. 583-589, 2016. 
[17] S. A. Shehzad, T. Hayat, and A. Alsaedi, "MHD flow of Jeffrey nanofluid with convective boundary conditions," Journal of the Brazilian Society of Mechanical Sciences and Engineering, vol. 37, no. 3, pp. 873-883, 2015.

[18] M. Sheikholeslami, M. Hatami, and D. D. Ganji, "Nanofluid flow and heat transfer in a rotating system in the presence of a magnetic field," Journal of Molecular Liquids, vol. 190, pp. 112-120, 2014.

[19] M. Mahmoodi and S. H. Kandelousi, "Kerosene-alumina nanofluid flow and heat transfer for cooling application," Journal of Central South University, vol. 23, no. 4, pp. 983-990, 2016.

[20] Z. Shah, T. Gul, A. M. Khan, I. Ali, and S. Islam, "Effects of hall current on steady three dimensional non-Newtonian nanofluid in a rotating frame with Brownian motion and thermophoresis effects," Journal of Engineering Technology, vol. 6, pp. 280-296, 2017.

[21] M. Sheikholeslami, M. Shamlooei, and R. Moradi, " $\mathrm{Fe}_{3} \mathrm{O}_{4^{-}}$ ethylene glycol nanofluid forced convection inside a porous enclosure in existence of Coulomb force," Journal of Molecular Liquids, vol. 249, pp. 429-437, 2018.

[22] M. Sheikholeslami, "Numerical investigation of nanofluid free convection under the influence of electric field in a porous enclosure," Journal of Molecular Liquids, vol. 249, pp. 12121221, 2018.

[23] M. Sheikholeslami and H. B. Rokni, "Numerical simulation for impact of coulomb force on nanofluid heat transfer in a porous enclosure in presence of thermal radiation," International Journal of Heat and Mass Transfer, vol. 118, pp. 823-831, 2018.

[24] Z. Shah, E. Bonyah, S. Islam, W. Khan, and M. Ishaq, "Radiative MHD thin film flow of Williamson fluid over an unsteady permeable stretching sheet," Applied Nanoscience, vol. 4, no. 10, pp. 825-830, 2018.

[25] A. Munir, S. Towers, A. Shahzad, and M. Khan, "Mass media and the contagion of fear: the case of Ebola in America," PLoS One, vol. 10, no. 6, pp. 1-13, 2015.

[26] A. Rehman, T. Gul, and Z. Salleh, "Effect of the Marangoni convection in the unsteady thin fico spray of CNT nanofluids," Processes, vol. 7, no. 6, p. 392, 2019.

[27] A. Rehman, Z. Salleh, T. Gul, and Z. Zaheer, "The impact of viscous dissipation on the thin film unsteady flow of GO-EG/ GO-W nanofluids," Mathematics, vol. 7, no. 7, p. 653, 2019.

[28] T. Gul, M. Z. Ullah, A. K. Alzahrani, and I. S. Amiri, "Thermal performance of the graphene oxide nanofluids flow in an upright channel through a permeable medium," IEEE Access, vol. 7, pp. 102345-102355, 2019.

[29] T. Gul, W. Noman, M. Sohail, and M. A. Khan, "Impact of the Marangoni and thermal radiation convection on the graphene-oxide-water-based and ethylene-glycol-based nanofluids," Advances in Mechanical Engineering, vol. 11, no. 6, 2019.

[30] T. Gul, H. Anwar, M. A. Khan, I. Khan, and P. Kumam, "Integer and non-integer order study of the GO-W/GO-EG nanofluids flow by means of Marangoni convection," Symmetry, vol. 11, no. 5, p. 640, 2019.

[31] T. Gul and K. Firdous, "The experimental study to examine the stable dispersion of the graphene nanoparticles and to look at the $\mathrm{GO}-\mathrm{H}_{2} \mathrm{O}$ nanofluid flow between two rotating disks," Applied Nanoscience, vol. 8, no. 7, pp. 1711-1727, 2018.

[32] S. Liao, "An optimal homotopy-analysis approach for strongly nonlinear differential equations," Communications in Nonlinear Science and Numerical Simulation, vol. 15, no. 8, p. 2003, 2010.
[33] T. Hayat, T. Muhammad, S. A. Shehzad, and A. Alsaedi, "Three-dimensional boundary layer flow of Maxwell nanofluid: mathematical model," Applied Mathematics and Mechanics, vol. 36, no. 6, pp. 747-762, 2015.

[34] Z. Shah, T. Gul, S. Islam et al., "Three dimensional third grade nanofluid flow in a rotating system between parallel plates with Brownian motion and thermophoresis effects," Results in Physics, vol. 10, pp. 36-45, 2018.

[35] Z. Shah, S. Islam, T. Gul, E. Bonyah, and M. Altaf Khan, "The electrical MHD and Hall current impact on micropolar nanofluid flow between rotating parallel plates," Results in Physics, vol. 9, pp. 1201-1214, 2018. 